\title{
Costa Rica sin Fronteras
}

En esta sección se publican los resúmenes en español de artículos que han sido publicados por investigadoras/es de universidades costarricenses en otras revistas en el mundo, con su debida referencia al trabajo original, y con una breve explicación de dónde se realizó la investigación. Los artículos originales han sido publicados en otros idiomas; las revistas tienen consejo editorial y manejan un proceso de revisión por pares.

Los resúmenes corresponden a estudios que se conforman a los criterios generales de la revista, esto es, se trata de "... estudios experimentales o que hagan recomendaciones concretas para solucionar problemas o preguntas relevantes (...). trabajos originales o de meta-análisis.”

\section{No existe efecto del suministro de carbohidratos sobre el rendimiento en una prueba contra reloj de $16 \mathrm{~km}$ de ciclismo.}

De Jeukendrup, A. E., Hopkins, S., Aragón-Vargas, L. F., \& Hulston, C. (2008). No effect of carbohydrate feeding on 16 km cycling time trial performance. European Journal of Applied Physiology, 104(5), 831-837.

El objetivo de este estudio fue investigar los efectos sobre el rendimiento de la ingesta de carbohidratos durante el ejercicio de alta intensidad de aproximadamente 25 minutos de duración. Doce ciclistas varones, entrenados en larga distancia (edad 19-41 años; masa corporal $73.2 \pm 4.2$ kg; $\mathrm{VO}_{2}$ max $66.4 \pm$

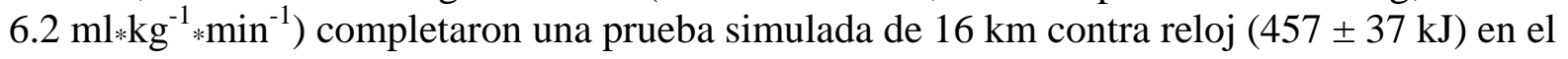
laboratorio en tres ocasiones. En una oportunidad, recibieron una solución de 6\% de carbohidratos y electrolitos (CHO), y en dos oportunidades recibieron la misma bebida placebo con electrolitos (PLA). Las bebidas de carbohidratos o placebo fueron ingeridas 5 min antes del inicio ( $4 \mathrm{ml} \mathrm{kg}^{-1}$ ) y a $25,50 \mathrm{y}$ $75 \%$ de completar la prueba contra reloj (1. $4 \mathrm{ml} \mathrm{kg}^{-1}$ ). La bebida CHO era una solución al 6\% de sacarosa-glucosa, y electrolitos. No se observaron diferencias entre los tratamientos en el tiempo para completar las pruebas contra reloj. Los tiempos en min:s fueron 25:30 \pm 1:34 y 25:27 \pm 1:46 para las dos condiciones placebo, y 25:38 \pm 1:59 para CHO. La potencia generada durante las pruebas contra reloj fue también notablemente parecida: $300 \pm 37 \mathrm{~W}, 301 \pm 39 \mathrm{~W}$ y $299 \pm 40 \mathrm{~W}$, respectivamente. Las estrategias de carrera y la frecuencia cardíaca fueron idénticas en las tres oportunidades. Se calculó un coeficiente de variación de $1.1 \%$ para esta prueba de rendimiento, de las dos pruebas placebo. Los resultados de este estudio aportan evidencia de que la ingesta de carbohidratos durante el ejercicio de alta intensidad y corta duración ( 30 min, 85-90\% $\mathrm{VO}_{2} \max$ ) no mejora el rendimiento. Además, este estudio encontró un coeficiente de variación muy bajo (1.1\%) para una prueba simulada de $16 \mathrm{~km}$ contra reloj.

Palabras clave: Nutrición deportiva. Confiabilidad. Deportes de resistencia. Bebidas deportivas.

Estudio realizado en la Universidad de Birmingham, Reino Unido. Colaborador: Luis Fernando Aragón Vargas.

La revista European Journal of Applied Physiology tiene un factor de impacto de 1.931. 


\section{Respuestas posprandiales de los triglicéridos al ejercicio aeróbico y niacina de liberación prolongada.}

De Plaisance, E. P., Mestek, M. L., Mahurin, A. J., Taylor, J. K., Moncada-Jiménez, J., \& Grandjean, P. W. (2008). Postprandial triglyceride responses to aerobic exercise and extended-release niacin. The American Journal of Clinical Nutrition, 88(1), 30-37.

Antecedentes: El ejercicio aeróbico y la niacina son utilizados frecuentemente como estrategias para reducir los triglicéridos en el suero sanguíneo; sin embargo no existe información relacionada al efecto combinado de estas estrategias en los triglicéridos posprandiales. Objetivo: Se compararon los efectos del ejercicio aeróbico y 6 semanas de la ingesta de niacina de liberación prolongada sobre los triglicéridos posprandiales en hombres con síndrome metabólico. Diseño: Quince participantes realizaron 4 condiciones experimentales: control - únicamente una comida alta en grasa (100 g grasa); ejercicio - ejercicio aeróbico ejecutado $1 \mathrm{~h}$ antes de la comida alta en grasa; niacina - comida alta en grasa consumida luego de 6 semanas de niacina; y niacina + ejercicio - comida alta en grasa consumida 1 h después de ejercicio aeróbico. Se midieron las respuestas temporales para las concentraciones de triglicéridos e insulina y se calcularon las áreas bajo la curva total $\left(\mathrm{AUC}_{T}\right)$ e incremental $\left(\mathrm{AUC} \mathrm{C}_{I}\right)$. Se determinaron las diferencias con análisis de varianza de 2 factores repetidos $(<0.05)$. Resultados: El ejercicio redujo los triglicéridos del $A U C_{I}$ en 32\% en comparación con el control (724 \pm 118 y $1058 \pm$ 137, respectivamente). La niacina no tuvo influencia en los triglicéridos $A U C_{I}$ y atenuó el efecto reductor del ejercicio sobre los triglicéridos cuando se combinaron. La combinación de niacina + ejercicio no tuvo efecto sobre los triglicéridos $A U C_{I}$ pero redujo el $A U C_{I}$ de la insulina luego de la administración de niacina. Conclusiones: El ejercicio aeróbico reduce la respuesta posprandial de los triglicéridos a una comida alta en grasas. La niacina reduce los triglicéridos en ayuno pero no los posprandiales y parece que afecta el efecto reductor del ejercicio cuando se consume combinado. Sin embargo, el ejercicio reduce las concentraciones de insulina posprandiales luego de la administración de niacina, lo cual indica el beneficio metabólico potencial del ejercicio en personas que ingieren niacina.

Palabras clave: Niacina, síndrome metabólico, ejercicio

Investigación realizada en el Departamento de Kinesiología de la Universidad de Auburn, Alabama, USA. Colaborador: José Moncada Jiménez.

La revista The American Journal of Clinical Nutrition tiene un factor de impacto de 6.6 en el Journal Citation Reports ${ }^{\circledR}$ del Institute for Scientific Information (Thomson Reuters, 2007). 
Pensar en Movimiento: Revista de Ciencias del Ejercicio y la Salud. Vol. 6, Nº1, 2008, ISSN 1659-4436

Costa Rica sin Fronteras

\section{Rehidatación después del ejercicio en el calor: una comparación de cuatro bebidas de uso común.}

De Shirreffs, S. M., Aragón-Vargas, L. F., Keil, M., Love, T. D., \& Phillips, S. (2007). Rehydration following exercise in the heat: a comparison of four commonly used drinks. International Journal of Sport Nutrition and Exercise Metabolism, 17(3), 244-258.

Para determinar la efectividad de 3 bebidas de uso común en la restauración del equilibrio de fluidos y electrolitos, 8 voluntarios se deshidrataron hasta 1.94\% $\pm 0.17 \%$ de la masa corporal, haciendo ejercicio intermitente en el calor, y luego ingirieron una solución de carbohidratos y electrolitos (Gatorade), una mezcla de jugo de manzana con agua carbonatada (Apfelschörle), o agua mineral San Benedetto en un volumen igual al 150\% de la pérdida de masa corporal. Todas estas bebidas tienen una percepción de ser soluciones efectivas para la rehidratación; su efectividad se comparó con la efectividad de rehidratación del agua mineral Evian, la cual no es percibida así por los atletas. Cuatro horas después de la rehidratación, los participantes estaban en un estado de hidratación significativamente menor que cuando se les evaluó al inicio en las condiciones de Apfelschörle ( $-365 \pm$ $319 \mathrm{~mL}, P=0.030)$, Evian $(-529 \pm 319 \mathrm{~mL}, P<0.0005)$, y San Benedetto $(-401 \pm 353 \mathrm{~mL}, P=0.016)$, pero estaban en el mismo estado de hidratación que antes del ejercicio deshidratante en la condición Gatorade ( $-201 \pm 388 \mathrm{~mL}, P=0.549$ ). El equilibrio de sodio fue negativo en todas las condiciones a lo largo del estudio; solamente con el Apfeschörle se mantuvo a los participantes en equilibrio positivo de potasio. En este contexto, la recuperación del equilibrio de fluidos sólo puede alcanzarse cuando se ingieren cantidades significativas, aunque quizás insuficientes, de sodio después del ejercicio. Existe un espectro limitado de productos comercialmente disponibles que tienen una composición suficiente como para alcanzar esto, a pesar de que el público piensa que algunas de las bebidas tradicionales son efectivas para este propósito.

Palabras clave: Equilibrio de fluidos, equilibrio electrolítico, hidratación, rehidratación, recuperación post-ejercicio.

Investigación realizada en la Universidad de Loughborough, Reino Unido. Colaborador: Luis Fernando Aragón Vargas.

La revista International Journal of Sport Nutrition and Exercise Metabolism tiene un factor de impacto de 1.438 para el 2008. 